\title{
Un lector crítico de educación inclusiva
}

\author{
Aldo Ocampo González*
}

\section{Centro De Estudios Latinoamericanos De Educación Inclusiva}

La enseñanza de la educación inclusiva no constituye un centro de análisis contingente por parte de los investigadores educativos y de las instituciones formadoras de educadores a nivel de pre y post-graduación. Un aspecto clave en la consolidación de una consciencia crítica sobre el sistema-mundo, requiere de la formación de un lector crítico de la realidad y de sus fenómenos, así como de procesos formativos que fortalezcan la construcción de sus significados asociados. Ambos constituyen poderosos procesos formativos de carácter hermenéutico que sólo acontecen en la interioridad de la micropráctica. La configuración de un 'método crítico' aplicado a la comprensión epistemológica de la educación inclusiva construye su actividad heurística a través de la determinación de aquello que leen sus obras y literatura, así como sus tecnologías de enmarcamiento; exigiendo atender a las condiciones de focalización que participan de sus principales interpretaciones. Al constituir el movimiento prefiero el término 'movimiento' en vez de paradigma o enfoque debido al déficit epistémicometodológico que ésta expresa y a la debilidad de sus sistemas de razonamientos para

* Director y fundador del Centro de Estudios Latinoamericanos de Educación Inclusiva (CELEI), Chile. Doctor en Ciencias de la Educación aprobado Sobresaliente mención 'Cum Laude', por la Universidad de Granada, España.

E-mail: aldo.ocampo.gonzalez@gmail.com 
develar su estatus ${ }^{1}$ - por la educación inclusiva un dispositivo reciente en la historia del pensamiento educativo, no se evidencian trabajos que con anterioridad aborden la pregunta por las tradiciones interpretativas de sus fenómenos. Tampoco se identifican con claridad y legitimidad académica propia un conjunto de reglas de interpretación que sus usuarios pueden aplicar en la lectura del mundo. Entonces, ¿cómo se enseña a leer el sistema-mundo en esta singular modalidad de encuadramiento teórico-político? Me interesa examinar las formas de construcción interpretativas dispuestas en la interioridad de las estructuras académicas y el tipo de modalidades didácticas que apoyan dicha empresa.

La designación 'lector crítico' es un concepto que se encuentra en lo más profundo del legado hermenéutico. Su actividad cognitiva no sólo queda sujeta a la recepción del significado que puede ser impuesto, ideológicamente o no, a través de su formación, sin que active las condiciones de producción de sus significados. Como operación intelectual bivalente queda compuesta por complejas formas de producción y recepción del significado. En tanto objeto de análisis, exploraré qué fuerzas se despliegan y colocan en juego en el espacio que constituyen ambas formas, puede ser útil recurrir analíticamente a la potencia de la 'y-cidad'2 propuesta por Bal (2018); es decir, la fuerza conjuntiva de ambos términos. Me interesa indagar en aquello que se ubica 'entre', que actúa como un nexo, una copula o una unión singular, entre las condiciones de elaboración y recepción del significado en los análisis de los fenómenos albergados bajo el signo de educación inclusiva, en tanto ejercicio formativo. Si bien es cierto,

\footnotetext{
${ }^{1}$ En la actualidad el estatus de la educación inclusiva no está claro. Diversas voces afirman que se trata de un paradigma, un enfoque o perspectiva; lo cierto es que su heurística desborda los problemas de un dominio o sub-dominio en particular; se interesa por abordar problemas educativos generales-estructurales de carácter complejo. La pregunta por el estatus a juicio de Ocampo (2019) explora, ¿qué es exactamente la educación inclusiva?, ¿es un concepto, un paradigma, una metáfora, un dispositivo heurístico, una metodología o bien, una teoría? Enmarcar la educación inclusiva a través de la noción de 'teoría' sugiere determinar cautelosamente que tipo de teoría designa. Sin duda, la educación inclusiva es un sistema de rearticulación de lo post-crítico.

${ }^{2}$ Múltiples usos y actuaciones de ' $y$ '. Corresponde al intersticio que articulan ambas nociones -sintagma educación inclusivaes regulado por un corpus de mecanismos de inter-implicación, da paso a un campo membránico, en el que, a pesar que ambas nociones presentan ideas similares, su especificidad diferencial es envuelta en un mismo espiral de análisis. En ella, son comprometidas las interioridades de ambas piezas, forjan una relación de mutualidad, un sistema de interioridad recíproca, "puesto que cada una está contenida en la otra como su alteridad" (Felman, 1977, p.6).
}

$\boldsymbol{\epsilon} \| 14$ 
el lector -en adelante, futuro educador- en el encuentro con el fenómeno, mediado por un conjunto de pre-entendimientos, no siempre actúa delante del fenómeno, sino detrás de él, es decir, de lo que queda. Situación similar sucede en el trabajo con determinadas formas paradigmáticas, teóricas y metodológicas. Las condiciones de recepción del significado, mayoritariamente, en la formación de los educadores se construyen a partir de lo que deja el paradigma, de la lectura de textos, de la consulta de diccionarios, de diálogos ya elaborados, nunca en dialogicidad crítica con el fenómeno, su práctica formativa deviene en una lectura evanescente del presente (Spivak, 2006). La formación de un lector crítico de los fenómenos educativos fortalece la competencia transversal referida al 'acto de leer'. Concebida como desempeño de comprensión, es decir, capacidad de demostrar flexiblemente lo aprendido e intervenir el mundo, las fuerzas de intimidad crítica ${ }^{3}$ desempeñan un papel preponderante. La formación de un lector crítico podría contribuir significativamente a subvertir las prácticas de oscurantismo y los dispositivos de ignorancias sancionadas que, comúnmente, vertebran las explicaciones educativas. ¿Cuáles son los textos que sancionan dicha empresa? Ninguno de los libros ampliamente difundidos como obras de referencia obligada en el terreno de la educación inclusiva, abordan la cuestión del lector crítico, ni tampoco inauguran un sistema de disposiciones específicamente intelectuales sobre su autenticidad epistemológica y de las rutas formativas que se desprenden de su naturaleza teórica.

Los procesos de elaboración y recepción de significados son producidos en un acto de co-creación. ¿Cómo son producidas sus interpretaciones y significados? La formación de los educadores debe agudizar condiciones de lectura minuciosa y recelosa de los objetos de análisis y espacios expositivos a los que se enfrenta al estudiantado, fortaleciendo su consciencia

\footnotetext{
${ }^{3}$ Ratifica un proceso de reinvención, "permite que el crítico utilice su propia relación íntima con la narrativa poética para informar sus lecturas de textos teóricos" (Bal, 2009, p.369). Su relevancia metodológica asume la "preocupación por mantener unido lo que sólo un estudioso separaría: la forma (lo que quiera que eso signifique), el contenido y el contexto; problemas a los que nos referimos como culturales, sociales o políticos" (Bal, 2009, p.368). La potencia heurística y analítica que encierra la noción de 'intimidad crítica' en tanto estrategia de análisis de las prácticas educativas, fomenta el intercambio constructivo con una multiplicidad de agenciamientos culturales y políticos, sujetos y territorios. Mediante el concepto de 'intimidad crítica' es posible cartografiar las formas culturales potencialmente despreciadas por la matriz colonial del saber, fortalece una escena de intercambios y diálogos, se opone abiertamente a las jerarquías pedagógicas y culturales legitimadas a través de los planteamientos liberales de la educación inclusiva. Se opone a las distancias, formas de marginación y subalternización de sujetos, agencias y formas de intervención y participación en el sistema-mundo.
} 
crítica, presencia en el mundo e intervención en éste. Tal dimensión formativa se consolida formando al futuro educador en contextos de lectura profunda de las patologías sociales crónicas ${ }^{4}$-endémicas en la constitución del sistema-mundo- que afectan a la enseñanza y se convierten en obstáculos complejos en el ejercicio del derecho a la educación. La lectura acerca de la heterotopicalidad de fenómenos ha de comenzar intencionando una ecología mental sobre las creencias que configuran parte del mundo cognitivo del estudiantado, opera en términos de un sistema de deconstrucción del conjunto de representaciones que ensamblan sus procesos de biografización y construcción de subjetividad, desempeñando un papel crucial en la fabricación y recepción del significado de determinadas problemáticas. La ecología mental se propone liberar el corpus de creencias -ya establecidas- para leer con éxito un determinado fenómeno. Educa la mirada interpretativa. La formación de un lector crítico de educación inclusiva se encuentra atenta a las reglas de interpretación que emergen por vía de su consolidación metodológica y objetual, las tradiciones interpretativas legitimadas en la diversidad de estructuras académicas, el estilo de determinaciones didácticas que serán implantadas en sus usuarios -futuros educadores-. Metodológicamente, cada uno de estos requerimientos, carecen de organicidad y desarrollo en la formalización de los estudios teóricos sobre educación inclusiva. La producción del significado en palabras de Culler (1998) siempre se encuentra determinada por el contexto, en tanto herramienta analítico-didáctica, no posee límites.

La educación del profesorado a través de la metáfora del 'lector crítico', inscribe su ámbito de discusión sobre el conjunto de condiciones que apoyan el recambio de significados sobre las imágenes y categorías que configuran sus ámbitos de inteligibilidad. Las condiciones de producción y recepción del significado afectan a la regulación ontológica ${ }^{5}$ de la diversidad de grupos albergados en las estructuras de escolarización convencionales o no-convencionales, regulan e inciden en la producción de un conjunto de signos que se imponen a cada colectivo, algunos reproducirán estratégicamente formas de devaluación y ausencia de reciprocidad, otros en cambio, impondrán categorías que llevan a una imperceptibilidad sistemática y

\footnotetext{
4 Dimensión endémica constitutiva de la estructura 'sistema-mundo'.

${ }^{5}$ Nivel de organización científica que en el contextualismo de la educación inclusiva adscribe a una política ontológica de lo menor, propia de la revolución molecular. Lo 'ontológico' en lo inclusivo es sinónimo de múltiples singularidades.
} 
silenciosa, otros impondrán condiciones de legibilidad y apertura del sujeto. Las dos primeras formas de regulación de la actividad sígnica impactan mediante condiciones cuya elaboración opera en la interioridad y en la sujeción del discurso y del sujeto. Exclusivamente, son objeto de articulación pasiva. La exterioridad, en cambio, fertiliza un espacio de apertura persistente, que evita cerrar la condición del sujeto a lo fijo, a lo estable, al poder de regulación del deseo social. Es la exterioridad la fuerza que niega la posibilidad de cerradura sobre sí mismo.

Retomando la insistencia de la pregunta didáctica acerca de los contextos, espacios y lugares dónde deben ser enseñados y leídos los textos y las problemáticas que constituyen el género ${ }^{6}$ denominado educación inclusiva, observo con fertilidad la cristalización de un espacio de 'intimidad crítica' en la formación pre y post-gradual de los educadores. Es aborrecible que se mantenga una misma práctica formativa, casi sin cuestionamiento alguno, en ambos ciclos. Las formas de abordar el objeto van mutando según la especificidad de las problemáticas y desafíos metodológicos dispuestos en la formación. La imperceptibilidad de este fenómeno, se debe a la ausencia de un método ${ }^{7}$ claro para formar a los educadores según las disposiciones

\footnotetext{
${ }^{6}$ El objeto de trabajo de la educación inclusiva impone 'otro lugar', es decir, al constituir un fenómeno de carácter postdisciplinar, forma un género singular en su constitución. Recurriendo al uso de las categorías identificadas por Genete (1989) en "Palimpsestos: la literatura en segundo grado", el género intelectual de la educación inclusiva bordea la especificidad de la transtextualidad y de lo transcientífico.
}

\footnotetext{
7 Véase el artículo: "Inclusión como método", Ocampo (2019). Inclusión como método presenta un singular enfoque analítico, se distancia de un conjunto de planteamientos que conciben la sección del sintagma referida al 'método' en tanto técnica o metodología en particular. Más bien, presenta la fabricación de una estrategia epistemológica para abordar una amplia variedad de problemas educativos de carácter complejos. La sección referida al 'método' configura una estrategia analítica y reflexiva destinada a examinar problemas y cuestiones que surgen de nuestras experiencias en la multiplicidad de microprácticas -herederas y reproductoras de una amplia variedad de micro-opresiones y micro-dominacionesentrecruzadas que ensamblan el sistema-mundo. Su heurística trabaja a partir de la metáfora post-colonial 'hablar de vuelta' que, a juicio de Smith (1999), configura un nuevo vocabulario y un sistema recognoscitivo dedicado a comprender una multiplicidad de problemáticas. La analítica que configura 'inclusión como método' puede asemejarse a lo que Mezzadra y Neilson (2012) denominan un nuevo continente de posibilidades políticas, éticas y epistemológicas, cuya espacialidad analítica e intensas preocupaciones e intereses, no responden a las lógicas de formalización de los métodos establecidos. Crea nuevas formas de lectura y mediación de los fenómenos que constituyen su campo de producción, análisis y lucha política. Forja una praxis crítica de examinación de la realidad, así como, de los principales proyectos de conocimiento que crean y garantizan la producción del conocimiento de la educación inclusiva. Es una analítica inquieta que no se cierra,
} 
del campo. La formación de un lector y un analista crítico del sistema-mundo se apoya, fundamentalmente, en la hermenéutica de la escucha y de la sospecha, con el objeto de visibilizar las estructuras ocultas del texto -acción social-y fenómeno. Las opciones formativas en este marco, demuestran que,

[...] la hermenéutica de la recuperación, al restringir el texto a un supuesto significado original ajeno a nuestras inquietudes, puede reducir su impacto; en cambio la hermenéutica de la sospecha valora el texto por la manera en que, sin que el autor sea consciente de ello, se compromete y nos ayuda a repensar temas de nuestro presente (incluso subvirtiendo, en este proceso, las ideas del autor) (Culler, 1998, p.85).

Un lector crítico sobre educación inclusiva se interesa por comprender los síntomas no-textuales y no tan evidentes implicados en la red de nudos que configuran la realidad examinada. Cada una de estas interpretaciones, a su vez, es parte de una actividad sígnica y escópica, es decir, mirada que cada época construye respecto de un determinado objeto de análisis. Su potencia heurística afecta a la legalidad de los signos y de sus significantes aceptados, regulan determinadas condiciones de conocimiento implícito en el encuentro con singulares contenidos o marcos conceptuales. La insistencia en la formación de un lector crítico devela, de qué manera, a través de la enseñanza de la educación inclusiva se construyen significados

ni detiene en los formalismos lógicos de ninguno de los repertorios metodológicos legados por las Ciencias Sociales. Es, también, un singular umbral analítico. La inclusión es, en sí misma, un dispositivo de transformación de la estructura de producción del conocimiento de la ciencia educativa en su conjunto. A este complejo proceso, en trabajos anteriores, he denominado dispositivo macro-educativo, que en sí mismo, es un sistema de recognición de la teoría educativa contemporánea. Coincidiendo con Chen (2010), las condiciones de producción son, en cierta medida, espacios imperceptibles de operación del colonialismo y el capitalismo, así como, del poder, específicamente, en fenómenos ambivalentes como el aquí analizado, que surge de complejos enredos genealógicos, así como, de la rearticulación de condiciones de producción de carácter extra e intra-teóricas. La capacidad heurística que contiene la noción de 'educación inclusiva', concibe la sección del sintagma 'inclusiva' como un punto de anclaje que posibilita la transformación de nuestra subjetividad, de allí que, Ocampo (2019) sostenga que ésta construye un singular estilo de subjetividad. La inclusión es, en sí misma, una propuesta crítica para transformar la estructura de conocimiento existente, al mismo tiempo, transforma la realidad y las consciencias de sus actores - principio de audibilidad-. Avanza en la construcción y/o producción diferente del conocimiento educativo, reconfigurando sus lenguajes, estructuras e ingenierías educativas. Se propone desenredar una variedad de estructuras de conocimiento que contribuyen a la reproducción imperceptible de una amplia variedad de expresiones del poder, así como, proyectos de conocimiento cómplices con la desigualdad, la opresión y la dominación.

E 18 
en relación a la examinación de determinadas problemáticas, o bien, cómo dan sentido a aquello que leen como parte de su actividad política, ética, heurística y didáctica. Emerge así, una preocupación que en el último tiempo ha guiado mi interés investigador, particularmente, orientado a comprender qué leen realmente los textos significados como obras de referencia obligatoria en la formación del profesorado en el contexto de la educación inclusiva. Sin duda alguna, esta es una tarea hermenéutica. Me interesa explorar los contornos de las prácticas y operaciones interpretativas que permitan dejar de pensar lineal y arbitrariamente sobre una amplia gama de problemas educativos, específicamente ligados al aseguramiento de condiciones del derecho 'en' la educación, la creación de condiciones de justicia a través de la enseñanza y del currículo, la detección de obstáculos complejos y otros ejes temáticos vinculados estrechamente a la educación de la multiplicidad de singularidades. En relación a la posibilidad de afirmar la presencia de tradiciones de interpretación propias de este campo, la respuesta no suele ser muy auspiciosa. Las prácticas de interpretación describen la variedad de contextos y condiciones en las que determinados objetos de análisis son compuestos e interpretados. De acuerdo con la especificidad diferencial de constitución del género de educación inclusiva, éstas no surgen de la articulación de su centro analítico-crítico.

Las formas de construcción del significado dependen en su mayoría del conjunto de experiencias previas y deseos del lector/analista ${ }^{8}$. Esto es lo que forma el horizonte de expectativas. ¿Qué papel desempeña la noción 'horizonte de expectativas' en la construcción de significado e interpretación de la multiplicidad de fenómenos que dan vida al contexto de análisis de la educación inclusiva? Culler (1998, p.80) sostiene que la examinación de un determinado fenómeno regula sus marcos y condiciones interpretativas, a partir de las respuestas que pueden ser ofrecidas ante un determinado foco de análisis. Singularidad de las variantes de análisis y el tipo de perspectivas que son inducidas, inciden significativamente en el funcionamiento del horizonte de expectativas. Si atendemos a las regulaciones del centro hegemónico de producción del conocimiento legitimado en la interioridad de las prácticas académicas institucionales, lo 'neo-especial' ${ }^{\text {' }}$, se instala como parte de la lectura

${ }^{8}$ Ambas categorías revisten la figura del estudiante/futuro educador.

${ }^{9}$ Corresponde a la estructura de conocimiento falsificada de educación inclusiva. Fundamentalmente, denota una operación de travestización e imposición del legado epistémico didáctico de Educación Especial para fundamentar lo inclusivo. 
del corpus de fenómenos que alberga, fundamentalmente, una perspectiva eurocéntrica, de carácter hegemónica cuya potencia actúa en términos de un mecanismo de ficción regulativa, imponiendo marcos del esencialismo -en sus diversas expresiones- y del individualismo metodológico. Su funcionamiento se aloja en el centro del capitalismo hegemónico, impone estratégicamente un ideal de emancipación y cambio en un conjunto de disposiciones normativas. ¿Es posible cambiar las maneras de leer los fenómenos educativos significados en el marco de la inclusión y la justicia educativa? Para alcanzar tal empresa, es necesario alterar la gramática epistemológica, interpretativa y formativa legitimada en las estructuras de formación -pre y post-graduación- del profesorado. Comprender a cabalidad lo que un determinado texto significa exige atender al contexto histórico en el que surgen sus condiciones de emergencia y mecanismos de legalidad. Toda interpretación es sancionada en la interioridad de un archivo singular de pensamiento.

La enseñanza de la educación inclusiva entiende que su punto de inflexión aplicada a cualquier titulación de pedagogía, inicia con la comprensión situada en el interior de la micropráctica acerca del funcionamiento de las diversas formas de injusticias, freno al autodesarrollo y opresión que tienen lugar en la cotidianeidad. Las condiciones de elaboración y recepción de los significados legalizados como parte de singulares fenómenos de análisis, se enseñan de cara al funcionamiento de la cotidianeidad. ¿Cómo enseña a leer sus fenómenos? ¿En qué se fundamenta su política interpretativa? Para responder a estas interrogantes, recurriré a la notable contribución de Young (2002). Su legado asegura cambios alterativos de carácter significativos en el fortalecimiento de la consciencia del futuro educador. Una práctica analítica asume las implicancias derivadas de movimientos, tales como, el feminismo, el postcolonialismo, la interseccionalidad, los movimientos de liberación de gente negra, gays, indígenas, etc., dominios significados como facilitaciones ausentes, es decir, excluidas y marginadas de las agendas intelectuales hegemónicas que problematizan el terreno y el género denominado educación inclusiva -específicamente, en su política ontológica-. De qué manera, las estructuras interpretativas empleadas -no vamos a decir legitimadas, ésta empresa resultaría de difícil acceso- en la formación del profesorado profundizan con rigor analítico los Ilamamientos -vacíos- de la justicia, la equidad, la democracia radical, etc. Los

Constituye uno de sus principales fracasos cognitivos, 'lo especial' y 'lo inclusivo' designan operaciones hipónimas, pero, diferentes a la vez.

$\boldsymbol{\epsilon} \mid 20$ 
núcleos principales de análisis que convergen en cualquier plan de formación de pre y postgraduación en el dominio de la inclusión, corresponden mayoritariamente al terreno de la filosofía política. ¿Tiene cabida esta dimensión en la educación del profesorado? En caso de tenerla, ¿cómo despliega su intelectualidad? ¿A qué concepciones de justicia, igualdad, ampliación de oportunidades, heterogeneidad del espacio, ciudadanía, democracia e inclusión conduce? En honor al espacio disponible por política editorial, la operación que articularé es la siguiente. En primer lugar, examinaré cómo enseñar la educación inclusiva a la luz de los aportes de Young (2002), y, posteriormente, intentaré responder al conjunto de interrogantes que preceden estas líneas. Tal vez, las respuestas que pueda ofrecer se ubican más próximas a acciones heterodoxas. Me interesa palpar analíticamente los contornos del reduccionismo político, ético y epistemológico que atraviesa nociones tales como justicia, inclusión, opresión, etc. El reduccionismo al que refiero interpela las prácticas de banalización intelectual que disfrazan lo diferente en la unidad y en lo colectivo.

Se enseña la educación inclusiva de cara a la comprensión multidimensional de la cotidianeidad, se enseña a reconocer cómo en determinadas circunstancias somos objetos de opresión, o bien, ejercemos dominación y poder sobre otros. Es una enseñanza situada en la micropráctica, en la transferencia hereditaria de códigos que se reproducen imperceptiblemente en nuestras interacciones diarias cada una de las patologías sociales crónicas, sus mascaradas y regulaciones performativas. Vista así, la enseñanza de la educación inclusiva y sus modalidades de teorización inscriben su actividad en términos de un dispositivo recognoscitivo. Observo necesario inaugurar las prácticas interpretativas, los espacios de concientización y la lectura de sus textos y fenómenos, a través de nociones, tales como, la opresión la injusticia, las formas de diferenciación social que son legitimadas imperceptiblemente en el espacio escolar y social y en singulares estilos de relaciones sociales, preferentemente. Un punto crítico clave por reparar en la formación de un lector crítico de la realidad educativa, desde una perspectiva de justicia recognitiva (Fraser y Honneth, 2006), asume el problema ontológico de los grupos sociales, es decir, agudizando las diferencias y aumentando los sistemas de devaluación social. Se enseña a leer el mundo para aprender a reconocer las trabas al auto-desarrollo -opresióny a la auto-determinación -dominación-, en nuestras interacciones familiares, sociales, culturales, laborales, afectivas, etc. Las restricciones referidas al autodesarrollo exigen marcos analíticos que permitan monitorear, de qué manera, determinados procesos institucionales sistemáticos impiden a las personas aprender a usar sus habilidades para satisfacer sus 
necesidades impactando en sus procesos de biografización y trayectorias sociales y escolares. Las restricciones centradas en la autodeterminación, focalizan sobre un corpus de condiciones institucionales que impiden que las personas sean autónomas en la determinación de sus acciones. Una de las principales limitantes que enfrenta la inclusión y la educación inclusiva reside en una estrecha concepción de bien común.

En este marco, los estudiantes deben comprender que los derechos constituyen bienes no-materiales, entonces, que significa distribuir un derecho centrado en la multiplicidad de singularidades. Generalmente, los textos empleados como literatura obligatoria en la formación inicial y continua de los educadores en el contexto de la inclusión, otorga una visión bastante reduccionista y estrecha en relación a los ejes de análisis de la filosofía política. Por ejemplo, se lucha por asegurar condiciones homogéneas para todos en materia de educación, lo que en cierta medida apoya la tesis universalizadora, a la vez, regula y agudiza el problema ontológico de los grupos sociales y las realidades escolares, traducida como escuelas que son mejores que otras, reclamando un dispositivo de dislocación que impone un signo de justicia redistributiva compleja, es decir, asegurar un mismo derecho atendiendo la especificidad y las necesidades de cada colectivo. De lo contrario, la arbitrariedad de las relaciones reproducirá sistemáticamente condiciones de aseguramiento de derechos ofertando a todos lo mismos. Es preciso recordar que, la política ontológica de la inclusión y las regulaciones de su espacio político se inscriben en la ontología de lo menor, en el giro molecular, en la monada, en la multiplicidad de diferencias. Las lecturas deben ayudar a los futuros educadores a entender el problema distributivo. La elaboración de nuevas formas de producción y recepción del significado apoyan la emergencia de nuevos efectos-de-legitimidad, efectos-de-sujeto y efectos-de-legibilidad. Las decisiones políticas deben amparase en la multitud.

La enseñanza de la educación inclusiva se convierte en un mecanismo de efectuación cuyo propósito persigue la elaboración de una crítica política de las opiniones sobre el sujeto, la opresión, la inclusión y la (in)justicia, preferentemente. Se lee el sistema-mundo con el afán de aprender a reconocer las operaciones imperceptibles que limitan el potencial transformativo de la inclusión, convirtiéndola en un problema técnico referida a la absorción de grupos significados como excluidos o marginados, se requiere un análisis que devele el funcionamiento de las estructuras de escolarización. Continuar operando desde la interioridad de los marcos epistemológicos tradicionales y sus categorías es, sin duda, responsable de la determinación 
de derechos, privilegios y obligaciones que benefician a unos cuantos ciudadanos, al tiempo que ponen en riesgo a otros muchos. Las prácticas interpretativas y la construcción de sus significados, se orientan para movilizar su frontera y socavar la opresión. Otro desafío consiste en aprender a gestionar los derechos según los diferentes grupos significados por raza, sexo, religión, clases social, ocupación etc. La enseñanza de la educación inclusiva orienta su aparato analítico a una comprensión relacional del derecho a la educación y de las formas en que imperceptiblemente articulan situaciones de exclusión y discriminación múltiple. La diferencia entendida como oposición instala un vector normativo que restringe la variación, la irrupción, la heterogeneidad y la multiplicidad. Si bien, el centro hegemónico de producción de la educación inclusiva reconoce que este es un campo de carácter relacional, a menudo tienden a reemplazar esta concepción en sus análisis por un examen diádico, caracterizado por designaciones, tales como, incluido/excluido, grupo mayoritario/grupo minoritario, víctima/ victimario, etc. En esta visión comprehensiva se pierde de vista el funcionamiento de una estructura más amplia de análisis que convergen en el estudio de las condiciones que crean y garantizan efectivamente diversos ejes de desigualdad estructural múltiple. En esta visión, las desigualdades múltiples y las formas de injusticias se entienden a través de un enmarcamiento distributivo, perdiendo de vista el fenómeno estructural que sostiene sus operaciones. Un examen estructural acerca de las condiciones de injusticia e inclusión, abandona el ideal de arbitrariedad focalizando sobre el conjunto de estrategias, previamente, intencionadas que restringen el desarrollo de una amplia variedad de personas. Un examen analítico sobre condiciones de injusticia a través de una política distributiva, se interesa por dar cuenta cómo se estructuran dichas condiciones de freno, al tiempo que "pierde de vista el modo en que los individuos poderosos instauran y reproducen su poder" (Young, 2002, p.58), insiste la politóloga norteamericana, comentando que,

[...] al conceptualizar el poder como relacional más que sustantivo, como producido y reproducido a través de mucha gente que está fuera de la diada inmediata del poder, se pone de manifiesto la naturaleza dinámica de las relaciones de poder como un proceso en marcha. Un análisis distributivo del poder oculta el hecho de que, como afirma Foucault, el poder existe solo en acción (Young, 2002, p.59). 
La enseñanza de la educación inclusiva sólo cobra valor en la medida que se articula relacionalmente comprometiendo su causa con una diversidad de significados. Desde un punto anti-esencialista, comprende que múltiples designaciones, entre ellas, la noción de grupos sociales, actúa en términos de un concepto ficticio, en el que

[...] la opresión ha sido perpetrada por una conceptualización de la diferencia de grupos en términos de naturalezas inalterables y esenciales que determinan lo que los miembros del grupo merecen o aquello de que son capaces, y que hacen que los grupos sean de tal modo excluyentes entre sí que no pueden tener similitudes ni atributos comunes. Para afirmar que es posible la existencia de diferencias de grupo social sin opresión es necesario conceptualizar los grupos de un modo mucho más relacional y flexible (Young, 2005, p.84-85).

¿Cuáles son los elementos centrales de la política interpretativa de la educación inclusiva? ¿Cómo los estudiantes llegan a tales interpretaciones? ¿De qué manera escogen entre estas? Como aspecto preliminar, Culler (1998) indica la necesidad de comprender los 'idiomas críticos' que sostienen la diversidad de procedimientos de lectura, con la intención de atender a aspectos, tales como, "lo lisible es aquello que responde a los códigos y sabemos cómo leer; lo scriptible es lo que resiste a la lectura y sólo puede ser escrito" (Culler, 1998, p.85). Un vector formativo pertinente, evita imponer un " "lector implicado» como a un papel simple que el lector está llamado a desempeñar, en la medida en que el placer bien puede llegar al lector, como dice Barthes, desde la interacción de compromisos contradictorios" (Culler, 1998, p.86). En tal caso, insiste el teórico estructuralista literario, señalando que, el "lector de llegar a conformar en el texto los códigos y convenciones consideradas relevantes y la resistencia del texto o su docilidad frente a las operaciones interpretativas particulares. La estructura y el significado de la obra emergen a través de una forma de la actividad del lector" (Culler, 1998, p.86). Las prácticas de lectura en la formación de los educadores a través del principio de 'intimidad crítica', devienen en un sistema de implicación dialógica con aquello que se lee, es un terreno de intertextos, de encuentros y acontecimientos que giran y dislocan la realidad y sus estados de consciencia. Es un signo de desbloqueos mentales. Se enseña inclusión con la finalidad de producir una ecología mental.

El lector que forma la educación inclusiva desde el centro hegemónico de regulación de lo neo-especial, es un practicante que consulta diccionarios, textos especializados, tejidos 
de voces elaboradas previamente sobre determinadas temáticas. Su habitabilidad adopta una posición insegura, interpela cómo se coloca al Otro en los marcos normativos y neoliberales que regulan la educación, cómo la diferencia lidia con la multiplicidad de problemáticas que afectan al desarrollo humano desde un punto de vista redistributivo, etc. La ética de la alteridad en este marco actúa en términos de una política de identidad, evita interrogar acerca de las posibilidades que residen en el sujeto para elegir o ejercer su agencia. El signo de inclusión que habita en la formación de los educadores mantiene una estrecha relación con la pauta política y cultural dominante, es una expresión alojada al interior del capitalismo supremacista, situación similar expresa el estatus de la justicia social y educativa, existe una cooptación de tales racionalidades. Interesa documentar de qué manera, a través de estas actuaciones, "qué subalterno queda estratégicamente excluido de la resistencia organizada" (Spivak, 2006, p.10). La formación de los educadores no se articula desde el hábito de leer el mundo, más bien, se reproduce la ingeniería educativa que no es, ni neo, ni tampoco liberal, obedece a una cuestión más extraña aún. La formación se convierte en un dispositivo crítico en la medida que es capaz de examinar las estructuras de producción de la razón de la inclusión y la justicia alojada al interior del capitalismo. En efecto, la tarea del formador del profesorado es asemejable a

[...] la tarea del profesor de análisis literario se sitúa en la aporía de una reorganización no coercitiva de la voluntad desde el momento en que estudiante y profesor oscilan entre la libertad-frente-a y la libertad-para; y no en la celebración de la voluntad de poder-de-clase estadounidense como resistencia no mediada (Spivak, 2006, p.11).

El discurso hegemónico sobre educación inclusiva -instalado de forma imperceptible y ortodoxa- al centrase exclusivamente en la educación de niños con discapacidades, impone un escenario pedagógico de la abyección y la reproducción imperceptible de la estructura de conocimiento falsificada de educación inclusiva y del saber neo-especial ${ }^{10}$, sirve a la

\footnotetext{
${ }^{10}$ Por neo-especial refiero a la empresa de lo especial que coopta la fuerza performativa de la inclusión en su dimensión auténtica, imponiendo su legado y consolidando una visión travestida y falsificada en torno al significante de la inclusión. De esta interpretación, es que surgen y se legitiman gran parta de los principios de regulación de dicho modelo al interior de las prácticas educativas. Se impone un signo de operaciones incesantes. Esta es una idea que debe ser forcluida de la formación de los educadores.
} 
reproducción de la desigualdad, del esencialismo estratégico y del individualismo metodológico. Este discurso se arma para operar estratégicamente en función de la creación de fuerzas de sujeción de la desigualdad y del modelo dominante, estableciendo una línea de continuidad al interior de las prácticas educativas. Sobre esto la deconstrucción puede resultar de ayuda. El sujeto educativo a través del giro epistemológico de la educación inclusiva se expresa mediante un conjunto de figuras que no pueden ser delimitadas en los marcos de las categorías tradicionales que ensamblan su discurso, evitando que sean estratégicamente excluidos del movimiento significado como inclusión y justicia educativa. La tarea crítica de la educación inclusiva consiste en superar sus limitaciones y sus automatismos de división. Los textos no logran articular una razón que 'cognoce' la naturaleza teórica de la educación inclusiva, de la justicia educativa y social, etc. Un aspecto a rescatar en la educación del profesorado consistirá en el rescate de su razón teórico-metodológica, hasta ahora coexiste en la interioridad de los programas de formación una razón falsificada y superficial que poco o nada dice en relación con el sentido auténtico de la matriz teórica del enfoque. En esta estructura, se impone una construcción de sujeto sin cognición de sí mismo, su plano interpretativo se aleja del signo de nuestra naturaleza humana.

¿Cuál es la disposición en sentido kantiano a la que nos conducen estos textos? Una respuesta posible refiere a la emergencia de un programa. Observo la configuración de un acceso programado que nos invita imperceptiblemente a trabajar en los contornos de una estructura falsificada de conocimiento y a la comprensión de determinados fenómenos. Este punto amerita una discusión a pie de página, pues corresponde al conjunto de presuposiciones que se legitiman imperceptiblemente en la dimensión formativa de los futuros profesores. Consecuentemente, esto repercute en un objeto falsificado. Se convierte en una fuerza que no tiene dominio sobre nosotros. ¿Es posible identificar una práctica de lectura de textos consolidada en la formación docente para analizar multiaxialmente, la diversidad de dominios y sus objetos específicos que desbordan sus límites y contornos? La verdad es que no.

Un aspecto interesante extraído desde la extensa obra de Spivak (2006) versa en torno a la idea de 'situación del aula', ese espacio inmaterial integrado por la capacidad narrativa de los propios hechos para contarnos, sensibilizarnos y hacernos ver el mundo a través de otros ojos, resulta clave en la articulación de un desbloqueo mental. En sí, la enseñanza de 
la educación inclusiva es un ejercicio de perturbación empática ${ }^{11}$, activa la figuratividad del fenómeno y la imaginación para destrabar sus problemáticas. La formación debe concebirse como un dispositivo que dispare la imaginación como vector escultórico del mundo. Las lecturas ofrecidas y los contenidos abordados contribuyen teórica y analíticamente a la comprensión del objeto -de carácter post-disciplinar-y de sus objetos específicos, y a su fortalecimiento. ¿Cómo se instrumenta la formación? Me interesa documentar de qué manera se forma un lector crítico. Una modalidad formativa con tales características, comenta Bal (2015) que, obliga al lector a detectar la naturaleza performativa de los pactos subjetivos, sus adherencias, empatías, acuerdos, rechazos, etc. a determinadas formas interpretativas en las que se cruzan y desbordan una complejidad de múltiples significados acerca de la experiencia de la exclusión, por ejemplo. Cómo se adaptan las situaciones de focalización presente/ausente y de acceso a la multiplicidad de situaciones que configuran el campo de lucha de la inclusión y su relación con la justicia. Se impone la pregunta por sus nuevos itinerarios intelectuales. El campo de educación inclusiva es un 'palimpsesto', comprende un territorio que hace patente el grabado de un conjunto de huellas y memorias epistémicas heterogéneas provenientes de diversos dominios intelectuales, éticos, políticos, discursivos y metodológicos. Es un grabado que opera en la performatividad de lo rearticulatorio, colaborando en la creación de otras nociones de análisis. El método de lectura e interpretación requerido en el contexto de la enseñanza de la educación inclusiva, se interroga, ¿̇la formación de los educadores obliga a leer los textos sobre inclusión como educadores?, o ¿cómo qué?

De acuerdo con Orlandi (2012), un lector o analista de educación inclusiva asume la necesidad de "cuestionar las maneras de leer, llevar al sujeto hablante o al lector a preguntarse sobre lo que producen y lo que oyen en las diferentes manifestaciones del lenguaje" (p.17) y de singulares procesos de orden cultural y social. Concebida así, designa una entrada en lo simbólico, cuyos signos alteran y modelizan el funcionamiento de la sociedad. "Saber cómo funcionan los discursos es colocarse en la encrucijada de un doble juego de la memoria: el

\footnotetext{
${ }^{11}$ Un sistema de 'perturbación empática' es "aquello que nos incómoda, perturba, frustra, su constitución oscila entre la implicación y la ruptura, nos reformula de manera productiva" (Hite, 2016, p.19). Insiste la investigadora norteamericana, agregando que, es "un terreno cognitivo y emocional que se mueve entre la implicación y la ruptura, la dinámica entre la transición y la recepción" (Hite, 2016, p.19).
} 
de la memoria institucional que estabiliza, cristaliza y, al mismo tiempo, el de la memoria constituida por el olvido, que es lo que torna posible lo diferente, la ruptura, lo otro" (Orlandi, 2012, p.18).

La 'inclusión' como categoría de análisis enfrenta un problema definitorio particular, participa de las relaciones de poder estructurales y de las representaciones culturales que interroga. Sin embargo, la comprensión de las condiciones que hacen su conocimiento comprensible desde la autenticidad del fenómeno, constituye junto a su objeto -el qué- y método -el cómo-, uno de sus puntos más álgidos y espinosos. Al reconocer que la inclusión participa de las relaciones que interpela, atiende al conjunto de acciones que la sitúan como reflejo de las relaciones de poder a nivel estructural y de su micro-práctica. La educación inclusiva como proyecto intelectual construye una estrategia de resistencia epistémica micropolítica epistémica y analítica-, analiza cómo determinados cuerpos de conocimiento se colocan al servicio de la dominación y opresión -por ejemplo, la empresa que alberga la propuesta del esencialismo liberal-, o bien, cómo el conocimiento influencia la configuración de las relaciones de poder (Hill Collins, 2015). Por norma, los sistemas de razonamientos hegemónicos significados como parte de la educación inclusiva, tienden a omitir un examen que aborde la inclusión situada dentro de las estructuras de poder y de las representaciones culturales, ambos temas clave en su vinculación con la justicia. Desconoce las formas de (re) articulación de los diversos discursos sobre inclusión, imponiendo un significante unívoco que pone en desmedro el encuentro con la multiplicidad de diferencias o singularidades múltiples, ni el conjunto de relaciones en las que se inscriben sus significados. La multiplicidad de expresiones del poder, constituyen dominios de opresión y dominación históricamente construidos, cuyo poder organiza a determinados grupos al interior de las estructuras de escolarización, invisibilizando la presencia de obstáculos complejos y tecnologías de cooptación de determinados estudiantes a las fronteras del derecho 'en' la educación.

La educación inclusiva en tanto estrategia analítica y política, articula su poder de intervención en el mundo, se emplea para abordar las diversas formas de desigualdad que estructuran los procesos de escolarización, especificando, cómo determinados saberes o proyectos políticos y educacionales contribuyen silenciosamente a reproducir desigualdad haciendo uso de algunos de los principios más elementales propuestos por este campo. Analiza críticamente, qué proyectos de conocimiento sirven a la desigualdad, la opresión y 
a la dominación. Sin duda, proliferan argumentos vinculados a la multiplicidad de formas del esencialismo y del individualismo metodológico, sistemas de razonamientos en los que el sujeto queda estratégicamente excluido de proyectos significados 'en resistencia'. La inclusión encuentra su campo de actividad más allá de un análisis mono-sistema -binarismo incluido/ excluido-, se propone introducir un mayor nivel de complejidad en el estudio de los problemas educativos, abandona el estudio de la desigualdad desde un punto de vista mono-categorial. Su razón, en parte, dedica su actividad analítica a examinar las formas sociales en las que cada formato del poder tiene lugar, sugiriendo un análisis más profundo, complejo y matizado, respecto las (re)articulaciones entre las estructuras sociales y las representaciones culturales (Hill Collins, 2015).

\section{Referencias}

Bal, M. (200). Conceptos viajeros en las humanidades. Una guía de viaje. Murcia: Cendeac.

Bal, M. (2015). “In the absence of Post-". Revista Papeles, Vol.6 (12)-7(13)12-25.

Bal, M. (2018). “Y-cidad: los múltiples sentidos de 'y'”. Versants, 65 (3), 187-207.

Culler, J. (1998). Breve introducción a la teoría literaria. Barcelona: Crítica.

Chen, K. (2010). Asia as method: towards deimperialization. Durham: Duke University Press.

Genete, G. (1989). Palimpsestos. La literatura en segundo grado. Madrid: Taurus.

Felman, S. (1977). «To Open the Question», Yale French Studies: Literature and Psychoanalysis. The Question of Reading: Otherwise, 55/56, 5-10.

Fraser, N., Honneth, A. (2006). ¿Reconocimiento o redistribución? Madrid: Morata.

Hill Collins, P. (2015). Intersectionality. As critical social theory. Duke: Duke University Press.

Hite, K. (2016). Pedagogía crítica, perturbación empática, y la política de los encuentros en los espacios de la memoria en Chile. Colección Signos de la Memoria. Santiago: Museo de la Memoria y los Derechos Humanos. 
Volumen 3 • Número 1 • enero - junio 2020 • Págs. 13 - 30 •

ISSN: 2389-8798 impreso / ISSN 2539-1445 (En línea) / Tunja, Boyacá (Colombia)

Mezzadra, S., Neilson, B. (2012). Border as Method. Durham: Duke University Press.

Ocampo, A. (2019). “Contornos teóricos de la educación inclusiva”. Revista Boletín Redipe, Vol. 8 (3), 66-95.

Orlandi, E. (2012). Análisis del discurso: principios y procedimientos. Santiago: LOM.

Smith, L. T. (1999). A descolonizar las metodologías. Investigación y pueblos indígenas. Santiago: LOM.

Spivak, G. (2006). Crítica de la razón poscolonial. Hacia una historia del presente evanescente. Madrid: Akal.

Young, I. (2002). La justicia y la política de la diferencia. Valencia: Cátedra.

$\boldsymbol{\epsilon} \mid 30$ 